\title{
ESTUDIO SOBRE LAS PASTAS DE URNAS NEGRO SOBRE ROJO TARDÍAS DE YOCAVIL (PCIA. CATAMARCA, NOROESTE ARGENTINO). UNA PRIMERA APROXIMACIÓN
}

\author{
STUDY OF CERAMIC PASTE OF BLACK ON RED LATE URNS \\ FROM YOCAVIL (CATAMARCA PROVINCE, NORTHWESTERN \\ ARGENTINA). A FIRST APPROACH
}

\section{Valeria Palamarczuk ${ }^{1}$, Alejandra Reynoso ${ }^{2}$ y Marina Marchegiani ${ }^{3}$}

1. CONICET, Museo Etnográfico "Juan B. Ambrosetti", Facultad de Filosofía y Letras, Universidad de Buenos Aires. Moreno 350 (1091) CABA, República Argentina. E-mail: valepala@yahoo.com.ar

2. Museo Etnográfico "Juan B. Ambrosetti", Facultad de Filosofía y Letras, Universidad de Buenos Aires. Moreno 350 (1091) CABA, República Argentina. E-mail: ad_reynoso@yahoo.com.ar;

3. Museo Etnográfico "Juan B. Ambrosetti", Facultad de Filosofía y Letras, Universidad de Buenos Aires. Moreno 350 (1091) CABA, República Argentina. E-mail: marinamarchegiani@yahoo.com.ar

Presentado el: 04/11/2013 - Aceptado 09/04/2014

\section{Introducción}

El propósito del trabajo es ofrecer una caracterización inicial de las pastas de las urnas Negro sobre Rojo tardías de Yocavil, también conocidas como Belén - Santa María o Santa María Negro sobre Rojo mediante el estudio petrográfico de una muestra de cortes delgados. Se trata de una variedad de tinajas con particularidades de diseño, forma y proporciones, en la que notamos una síntesis entre los estilos alfareros tardíos ${ }^{1}$ Belén y Santa María (Figura 1).

Los estilos Belén y Santa María son característicos de dos sectores diferenciados, aunque contiguos, en el área valliserrana del Noroeste Argentino. La alfarería Belén es uno de los estilos tardíos con decoración de baños y pinturas más abundantes en los conjuntos alfareros de las zonas de Hualfín, Andalgalá, Tinogasta y aledaños (Basile 2009; Quiroga y Puente 2007; Wynveldt 2009). La alfarería Santa María de la Tradición Yocavil, es característica de los valles de Yocavil, el Cajón, Tafí y el piedemonte oriental de la provincia de Tucumán (Caviglia 1985; Nastri 1999). 


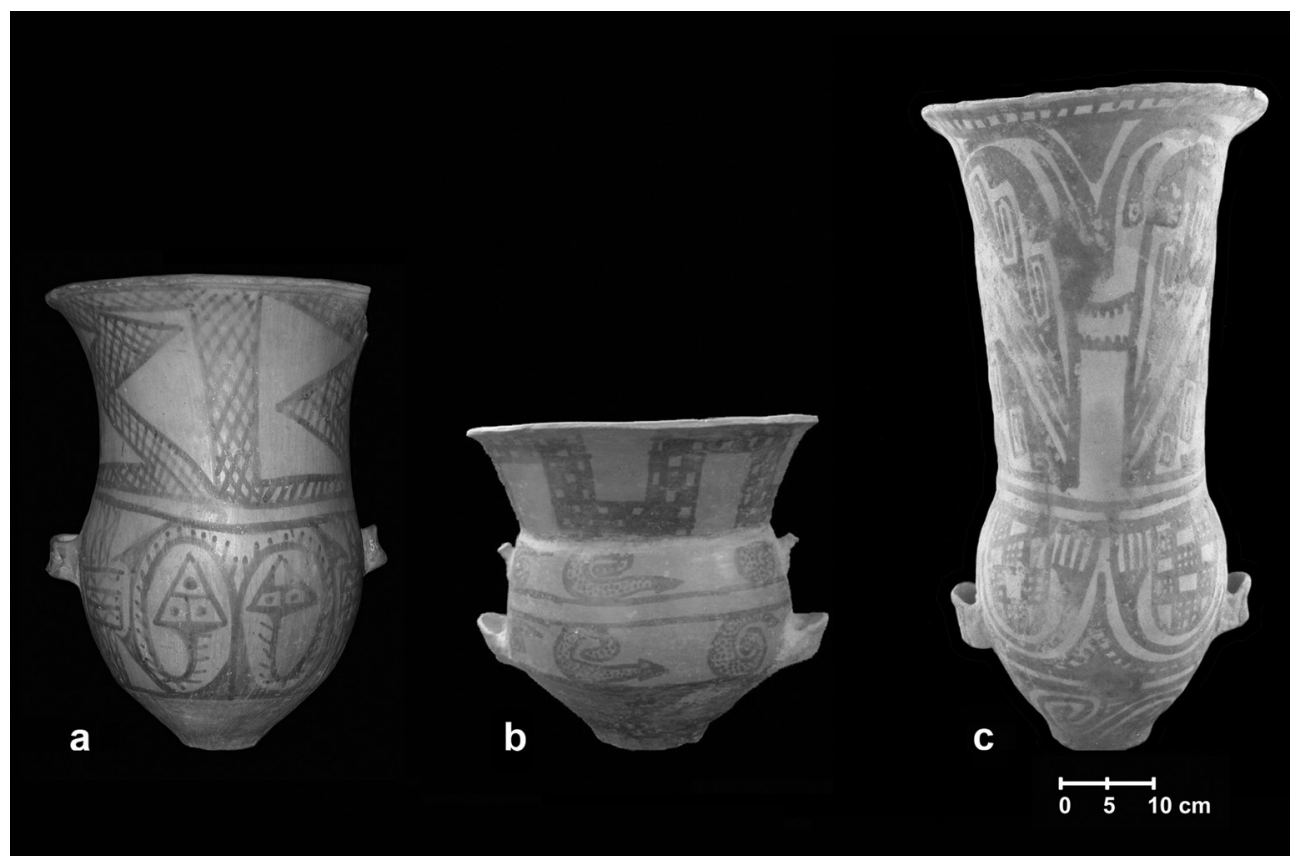

Figura 1. Ejemplos de urnas. a: Negro sobre Rojo tardía de Yocavil, Fuerte Quemado, Museo Etnográfico “Juan

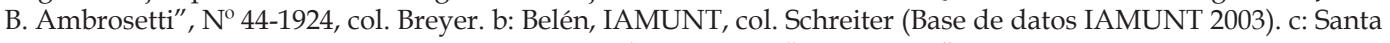
María, Rincón Chico, Museo “Eric Boman”.

Las alfarerías Santa María y Belén comparten expresiones estéticas, simbólicas y pautas de uso. Ambas producciones constituyen los estilos con baños y pinturas mayoritarios en sus respectivas áreas de referencia. Comparativamente la frecuencia de hallazgos de tinajas Negro sobre Rojo Belén - Santa María es muy baja. En base a una revisión bibliográfica y de colecciones de museos hemos identificado 43 ejemplares completos. Estas urnas comparten con las tinajas Belén el uso de pintura negra sobre un fondo rojo alisado o pulido, la aplicación de baño rojo en la superficie interna del cuello, la distribución de los campos decorativos, ciertos motivos y modos de representación. Por su parte, comparten con las tinajas Santa María bicolor rasgos como el predominio de cuerpos ovaloides, la tendencia al desarrollo de los cuellos largos, ciertos modos de representación de la serpiente y de "guerreros" en algunos ejemplares. A pesar del "equilibrio" de rasgos de ambos estilos en las urnas Negro sobre Rojo, no ocurre lo mismo con el ámbito geográfico en el cual circularon, siendo los hallazgos mucho más frecuentes en la zona de Yocavil y aledaños (Figura 2). El estudio de varios contextos de hallazgo indica que esta modalidad estuvo vigente posiblemente entre los momentos finales del Período Tardío, la época de expansión incaica en el Noroeste argentino y el Período Colonial Temprano (Marchegiani et al. 2009).

De manera muy general podemos decir que la cerámica Santa María bicolor de la Tradición Yocavil participa de la opción tecnológica del tiesto molido. Junto con los microtiestos, presentes en la mayoría de las vasijas, se podían agregar a las arcillas sedimentos arenosos en proporción variable (Palamarczuk 2002; Piñeiro 1996; Schwartz 1991). Por su parte el estilo Belén se separa de esta tendencia, pues las pastas poseen inclusiones arenosas, pero en su gran mayoría, carecen de microtiestos (Puente 2011; Zagorodny et al. 2010). El análisis tecnológico de las urnas Negro sobre Rojo tardías de Yocavil es materia que debe ser profundizada. Antecedentes para el valle de Tafí, en la provincia de Tucumán, mencionan 


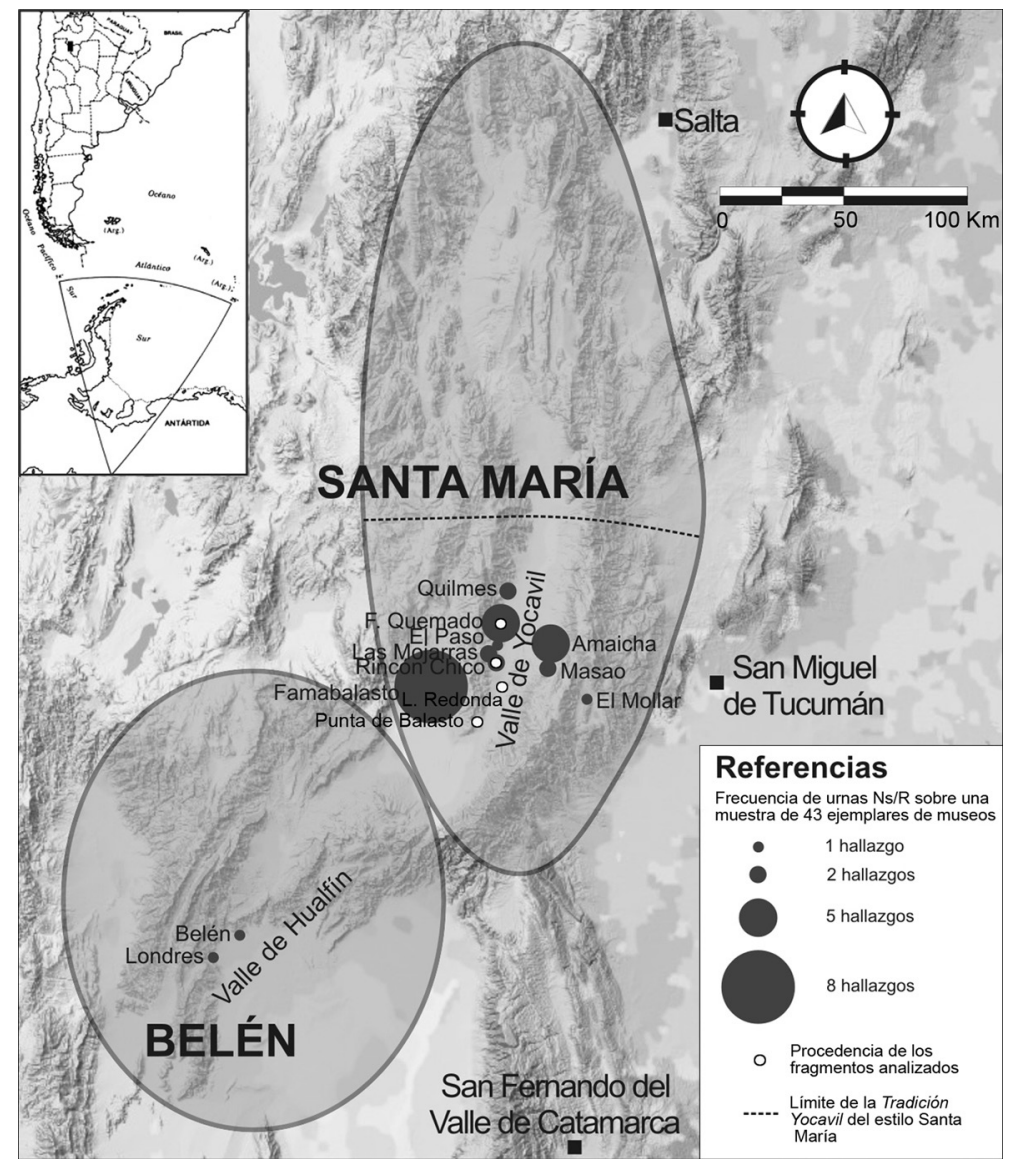

Figura 2. Distribución espacial de hallazgos de urnas Negro sobre Rojo en base a una muestra de 43 ejemplares de museos y ubicación de las procedencias de las muestras analizadas en este trabajo.

la existencia de abundantes inclusiones piroclásticas en las pastas de urnas "Santa María Negro sobre Rojo", semejantes a las observadas en alfarerías incaicas de la región (Páez y Arnosio 2009). En esta nota presentamos la primera caracterización de las pastas de esta variante en base a fragmentos obtenidos en la región de Yocavil, provincia de Catamarca, incorporando una línea de evidencia en torno a la manufactura de estas vasijas para aportar a la discusión de los ámbitos productivos y de los posibles actores sociales involucrados en su producción.

\section{Objetivos}

El objetivo principal es ofrecer una caracterización inicial de las pastas cerámicas de urnas Negro sobre Rojo tardías de Yocavil o Belén - Santa María mediante el estudio petrográfico de secciones delgadas. En base a esta descripción se comenzará a comparar las tendencias tecnológicas de este conjunto "mixto" con los estilos tecnológicos (Lemmonier 1992) o modos de hacer, que definen a las cerámicas Belén y Santa María. Esta comparación, basada en esta instancia en información bibliográfica, busca establecer en qué medida la integración de rasgos morfológicos y de diseño de ambos estilos, que apreciamos en aspectos 
"visibles" sensu Puente (2011) de las urnas Negro sobre Rojo tiene un correlato en aquellos rasgos tecnológicos "no visibles", como por ejemplo las clases de inclusiones presentes y de antiplásticos empleados.

\section{Método}

Debido a que las urnas Negro sobre Rojo tardías de Yocavil son una modalidad minoritaria, la obtención de fragmentos adecuados plantea dificultades. Para esta primera etapa se reunieron 6 fragmentos que poseen las dimensiones y conservan los rasgos diagnósticos suficientes para el reconocimiento sin ambigüedades de la variante. Proceden de sitios de Yocavil: El Calvario de Fuerte Quemado (1), Rincón Chico (2), Loma Redonda (1) y Punta de Balasto (2) (Figura 2). Con excepción del fragmento de Loma Redonda, obtenido en la superficie de un área funeraria, los restantes provienen de contextos domésticos o productivos en asentamientos locales o instalaciones incaicas, y fueron recuperados por las autoras y colegas del Proyecto Arqueológico Yocavil a lo largo de diferentes temporadas de trabajo de campo ${ }^{2}$

Los cortes se realizaron de manera perpendicular a las superficies. La frecuencia relativa de los componentes (matriz, poros e inclusiones) se estimó mediante el conteo de 300 puntos empleando ocular grillado. Las inclusiones se describen de acuerdo al tipo, frecuencia relativa, tamaño, desgaste, orientación y selección (Orton et al. 1997). La identificación de tiesto molido se realizó de acuerdo con parámetros establecidos en descripciones sistemáticas y estudios experimentales (Cuomo Di Caprio y Vaughan 1993; Whitbread 1986; Reedy 2008). ${ }^{3}$ Para establecer rangos de tamaño de las inclusiones y poros se midieron un promedio de 70 unidades por corte. Se consideró de manera arbitraria un tamaño mínimo de las inclusiones en $0,04 \mathrm{~mm}^{4}$. Los poros se describieron por su forma y tamaño, observando la presencia de rellenos. En relación a la matriz se realizaron observaciones de coloración, translucidez y estructura (Courtois 1976). ${ }^{5}$

\section{Resultados}

En todos los casos la atmósfera de cocción es oxidante completa, observándose un núcleo gris en la muestra 6 . El porcentaje total de inclusiones varía en un rango entre 15 y $22 \%$. El porcentaje de poros varía entre 5,67 y 13,67\% (Tabla 1). Éstos son esféricos, aplanados e irregulares, muchos de ellos, interconectados y algunos presentan rellenos secundarios de

\begin{tabular}{|c|l|c|c|c|c|c|}
\hline Muestra & \multicolumn{1}{|c|}{ Localidad } & Poros & Matriz & Inclus. & $\begin{array}{c}\text { Características } \\
\text { de la Matriz }\end{array}$ & $\begin{array}{c}\text { Orientación } \\
\text { Inclusiones }\end{array}$ \\
\hline 1 & Punta de Balasto & 10,00 & 68,00 & 22,00 & A-L; SL-M; B & no \\
\hline 2 & El Calvario de Fuerte Quemado & 11,67 & 73,33 & 15,00 & A-L; SL-M; BB & algo \\
\hline 3 & Loma Redonda & 11,33 & 72,00 & 16,67 & A-L; SL-M; BB & no \\
\hline 4 & Rincón Chico (RCh 8$)$ & 13,67 & 66,67 & 19,67 & A-L; SL-M; BB & algo \\
\hline 5 & Rincón Chico (RCh 15$)$ & 6,67 & 75,67 & 17,67 & A-L; SL-M; B & algo \\
\hline 6 & Punta de Balasto & 5,67 & 72,67 & 21,67 & A-L; M; BB & poco \\
\hline
\end{tabular}

Tabla 1. Cuantificación de los componentes en los cortes petrográficos analizados. A-L: arcillo-limosa; SL: seudolepidoblástica; M: microgranosa; B: birrefringente; BB: baja birrefringencia. 
calcita (corte 3) o de un material de baja birrefringencia, posible sílice (corte 5). En todos los casos la matriz es de tipo arcillo-limosa, con estructuras de fondos de pasta de tipo seudolepidoblástica y algo microgranosa (1 a 5 ) y microgranosa (6), notándose casos de matrices con birrefringencia importante ( 1 y 5$)$.

En todos los cortes se identificó tiesto molido, en proporciones variables entre 9 y $18 \%$, siendo siempre la inclusión mayoritaria (Tabla 2). El rango de tamaño de esta inclusión va de muy fino hasta muy grosero, con clastos de hasta 2,33 mm (muestra 4). Se aprecian ejemplares de tiesto de segunda generación, incluido al interior de una inclusión mayor de tiesto molido (muestra 3), y también, tiestos procedentes de diferentes vasijas en una misma muestra (muestras 1, 3, 5 y 6). Las inclusiones minerales presentes en diferente proporción son: cuarzo, plagioclasa, biotita, muscovita, y feldespato potásico. Con excepción del corte 3 , se observaron clastos líticos de tipo metamórfico (esquisto) en baja proporción, siendo los

\begin{tabular}{|c|c|c|c|c|c|c|c|c|c|c|}
\hline & & & & & & & & & \multicolumn{2}{|c|}{ Clastos Líticos } \\
\cline { 8 - 13 } Muestra & Localidad & Qz & Plagio. & Vidrio & Muscov. & Biotita & Feld. K & Tiesto & Metam. & Granito \\
\hline 1 & PB & 0,33 & 0,67 & & & 1,00 & & 18,00 & 2,00 & \\
\hline 2 & CFQ & 1,33 & 1,67 & 0,33 & 0,33 & 0,33 & & 9,67 & 1,33 & \\
\hline 3 & LR & 5,33 & 1,67 & & & 0,33 & & 9,33 & & \\
\hline 4 & RCh & 4,00 & 0,67 & 0,33 & 0,33 & 0,33 & & 12,67 & 1,33 & \\
\hline 5 & RCh & 0,67 & 0,67 & & 1,00 & 0,67 & 1,00 & 11,33 & 1,33 & 1,00 \\
\hline 6 & PB & 4,00 & 2,67 & & 4,00 & & 1,33 & 9,00 & 0,67 & \\
\hline
\end{tabular}

Tabla 2. Cuantificación de las inclusiones. Qz: cuarzo; Plagio: Plagioclasa; Feld. K: Feldespato potásico; Metam: metamórficos.

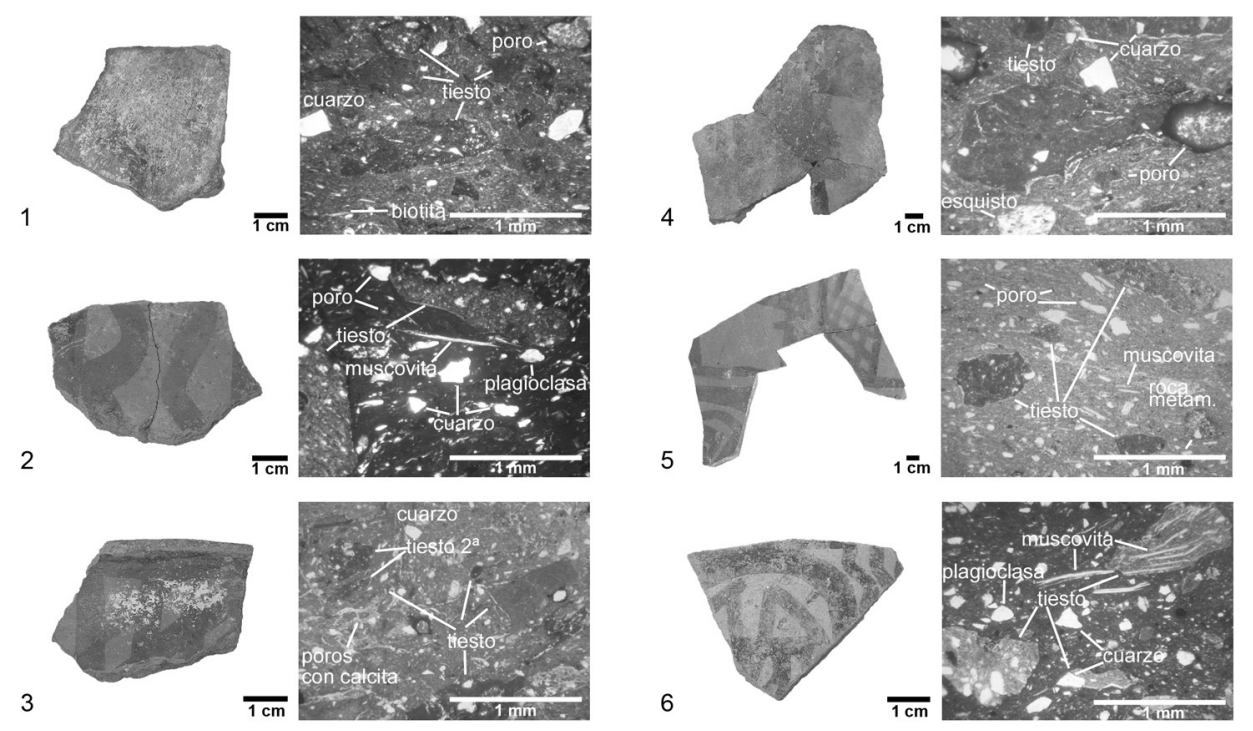

Figura 3. Fotografías de los fragmentos analizados. 
clastos de similar tamaño en las muestras 2, 4 y 5 y más pequeños en la 6 . El corte 5 posee escasos clastos graníticos y el número 2 y 4 escasas trizas vítreas (Figura 3). Las inclusiones minerales y líticas resultan consistentes con la geología regional (Ruiz Huidobro 1972; González et al. 2000) y presentan diversos grados de desgaste, pudiendo ser, en buena medida, antiplásticos agregados a la arcilla.

\section{Conclusiones}

La presencia de tiesto molido en elevada proporción en todos los casos (en porcentajes que entran en el rango de los reportados para vasijas de estilo Santa María) es indicativa de su incorporación intencional. Este modo de hacer vincula a los ejemplares observados con la tradición tecnológica del tiesto molido, de la cual participa el estilo Santa María, más no el Belén. Las características de los poros, el rango de tamaño de las inclusiones y la estructura de la matriz son también afines a ejemplares descriptos de estilo Santa María. El estudio petrográfico permite observar que los ejemplares analizados de esta variante mixta, en la que apreciamos la intensión de una fusión de las estéticas Belén y Santa María, se acercan, desde el aspecto de la producción de sus pastas, a los modos de hacer observados en la alfarería santamariana, lo que permite plantear como hipótesis la intervención de alfareros de Yocavil en su producción. Estas observaciones aportan una línea de información interesante para seguir reflexionando sobre las dinámicas sociales relacionadas al desarrollo de estilos mixtos. En este caso, hemos planteado en un trabajo anterior la posibilidad de que las urnas Negro sobre Rojo hayan sido el producto de una interacción social redefinida entre grupos de Hualfín y Yocavil, resultado a su vez de la configuración de ambas regiones como frontera del proceso expansivo incaico (Marchegiani et al. 2009).

La muestra considerada no es aún suficiente para vislumbrar la variabilidad tecnológica del grupo estilístico de las urnas Negro sobre Rojo, no obstante, este primer paso nos permite ver una opción tecnológica constante: la inclusión de tiesto molido. Será importante ampliar la muestra de fragmentos de Yocavil y observar fragmentos de esta particular variante de urnas que procedan del ámbito Belén, como así también, efectuar comparaciones directas con muestras de material Santa María y Belén. Están en proceso de análisis nuevos cortes que permitirán dar cuenta de ciertos aspectos de dicha variabilidad.

Agradecimientos: Al Lic. Eduardo Palamarczuk por la colaboración en el análisis de los cortes petrográficos. Proyectos UBACyT IJ 0064 y PICT 0113.

\section{Notas}

1. El apelativo "tardío" nos remite aquí a un bloque temporal que incluye los tradicionales Período Tardío, Inca y Colonial Temprano. Ambos conjuntos estilísticos, Belén y Santa María, fueron producidos, con variaciones, a lo largo de los períodos mencionados.

2. La metodología de cuantificación según familias de fragmentos (Orton et al. 1997) de los conjuntos cerámicos de los cuales se seleccionaron los fragmentos que componen la muestra garantiza que no haya repetición de análisis sobre ejemplares que pudieron pertenecer originalmente a una misma vasija.

3. La diferenciación entre tiesto molido e inclusiones arcillosas accidentales o incorporadas de modo voluntario puede plantear dificultades, en particular, cuando se trata de inclusiones aisladas y muy pequeñas. En los cortes analizados, esta dificultad no se presentó, 
debido a la abundancia de tiestos de tamaño grande en todos ellos, que exhiben semejanzas estructurales, de coloración, densidad óptica, etc. con microtiestos en el rango de dimensión problemática. En estos casos, la identificación de las inclusiones muy pequeñas como tiesto molido obedece a una apreciación contextual en el conjunto del corte.

4. Esta medida mínima se estableció en función de las posibilidades instrumentales para lograr una adecuada identificación del tipo de inclusión.

5. Determinaciones realizadas por el Lic. Eduardo A. Palamarczuk empleando microscopio Standard 18 Pol Zeiss.

\section{Bibliografía citada}

Base de datos de la colección arqueológica del Instituto de Arqueología y Museo, Universidad Nacional de Tucumán. Fundación Tiempos. Fundación CEPPA. Tucumán, octubre de 2003.

Basile, M.

2009. Recorriendo trazos. Un aporte a la definición de estilo decorativo Belén. Arqueología 15: $13-40$.

Caviglia, S. E.

1985. Las urnas para niños de los valles Yocavil y Calchaquí: su reinterpretación sobre la base de un enfoque gestáltico. MS.

Courtois, L.

1976. Examen au microscope petrographique des ceramiques archeologiques. Notes et Monographies Techniques $\mathrm{N}^{\circ}$ 8, Centre de Recherches Archeologiques. CNRS, Paris.

Cuomo Di Caprio, N. y S. J. Vaughan

1993. An experimental Study in Distinguishing Grog (Chamotte) from Argiliceous Inclusions in Ceramic Thin Sections. Archeomaterials 7: 21-40.

González, O. E.; M. E. Viruel, R. Mon, P. Tchilinguirian y E. Barber 2000. Hoja Geológica 2766-II San Miguel de Tucumán. SEGEMAR, Secretaría de Energía y Minería, Boletín $\mathrm{N}^{\circ} 245$. Buenos Aires.

Lemmonier, $\mathrm{P}$.

1992. Elements for an Anthropology of Technology. Museum of Anthropology, Anthropological Papers No 88. University of Michigan, Ann Arbor.

Marchegiani, M.; V. Palamarczuk y A. Reynoso

2009. Las Urnas Negro sobre Rojo Tardías de Yocavil (Noroeste Argentino). Reflexiones en torno al estilo. Boletín del Museo Chileno de Arte Precolombino, Vol. 14 № 1: 69-98.

Nastri, J. H.

1999. El estilo cerámico santamariano de los Andes del sur. Baessler-Archiv. Beiträge zur Völkerkunde, Neue Folgue, Band XLVII (LXXII.Band), Verlag von Dietrich Reimer, Berlin.

Orton C. P., P. Tyers y A. Vince

1997. La cerámica en Arqueología. Ed. Crítica, Barcelona. 
Páez, M. C. y M. Arnosio

2009. Inclusiones piroclásticas en pastas cerámicas del valle de Tafí: Implicancias para las prácticas de producción. Estudios Atacameños No 38: 5-20.

Palamarczuk, V.

2002. Análisis cerámico de sitios del bajo de Rincón Chico. Valle de Yocavil, Provincia de Catamarca. Tesis de Licenciatura en Cs. Antropológicas, orientación Arqueología, FFyL, UBA, MS.

Piñeiro, $M$.

1996. Manejo de recursos y organización de la producción cerámica en Rincón Chico, Catamarca. Relaciones de la Sociedad Argentina de Antropología, T. XXI: 161-185. Buenos Aires.

Puente, V.

2011. Prácticas de producción alfarera en el Valle del Bolsón (Belén, Catamarca). Materias primas y modos de hacer Ca. 900-1600 D.C. Tesis para optar al grado de Doctor en Filosofía y Letras, UBA. MS.

Quiroga, L. y V. Puente

2007. Imagen y percepción: Iconografía de las urnas Belén. Colección Schreiter. En: Procesos sociales prehispánicos en el sur andino. La vivienda, la comunidad y el territorio:323-346, A. E. Nielsen, M. C. Rivolta, V. Seldes, M. M. Vázquez y P. H. Mercoli Eds. Editorial Brujas. Córdoba.

Reedy, Ch. L.

2008 Thin-section petrography of Stone and ceramic cultural materials. Archetype Publications, Londres.

Ruiz Huidobro, O. J.

1972. Descripción geológica de la hoja 11e, Santa María. Servicio Nacional Minero Geológico, Boletín $\mathrm{N}^{\mathrm{o}} 134$, Buenos Aires.

Schwartz, G.

1991. Nuevos estudios sobre la cerámica Santa María (NOA). Primeros resultados de los análisis petrográficos, físicos y químicos. $47^{\circ}$ Congreso Internacional de Americanistas, Nueva Orleans.

Whitbread, I. K.

1986. The characterisation of argillaceous inclusions in ceramic thin sections. Archaeometry 28,1: 79-88.

Zagorodny N., Morosi M., Iucci M. E. y Wynveldt F.

2010. Estudios composicionales de las pastas de cerámica tardía del Valle de Hualfín (Belén, Catamarca). Arqueología No 16: 125-149. 